\title{
ChemComm
}

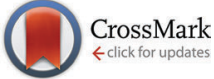

Cite this: Chem. Commun., 2015, 51, 7305

Received 3rd March 2015 Accepted 18th March 2015

DOI: $10.1039 / \mathrm{c} 5 \mathrm{cc} 01811 \mathrm{k}$

www.rsc.org/chemcomm

\section{4-Phosphopyrazol-2-yl alanine: a non-hydrolysable analogue of phosphohistidine $\dagger$}

\author{
Matthew Lilley, ${ }^{a b}$ Bezaleel Mambwe, ${ }^{b}$ Mark J. Thompson, ${ }^{a}$ Richard F. W. Jackson*a \\ and Richmond Muimo*b
}

\begin{abstract}
We report the synthesis of a stable analogue of $\tau$-phosphohistidine: 4-phosphopyrazol-2-yl alanine (pPza). Polyclonal antibodies generated against the mimic show high reactivity and selectivity for $\tau$-phosphohistidine, with minor or no cross-reactivity towards non-phosphorylated histidine or O-phosphoamino acids, including phosphotyrosine.
\end{abstract}

Protein phosphorylation occurs on several amino acid residues: serine, threonine, tyrosine, histidine, lysine, arginine, aspartate, glutamate and cysteine. ${ }^{1}$ It regulates most cellular processes and its dysregulation is linked to a number of diseases including cancer, diabetes, and hypertension amongst others. ${ }^{2}$ The $O$-phosphorylated amino acids (phosphoserine, phosphothreonine and phosphotyrosine) are extensively reported but only account for approximately $50 \%$ of phosphorylation events in mammalian cells, indicating alternative phosphoamino acids (histidine, lysine, arginine, aspartate, glutamate and cysteine) are abundant but understudied. ${ }^{3}$

Phosphorylation of histidine was first identified 50 years ago by Boyer and co-workers, ${ }^{4}$ and occurs on either of the two nitrogens located on its imidazole ring; accordingly, phosphorylated histidine exists either as $\pi$ (pros)-phosphohistidine 1 or $\tau$ (tele)-phosphohistidine 2 (Fig. 1), which is the dominant isomer found in proteins. It is now well established that phosphorylation of histidine regulates important cellular functions via the two-component phospho-relay signalling pathways in bacteria, fungi, and plants. Increasing evidence suggests phosphohistidine is also involved in regulation of key cellular processes in mammalian cells including ion channels, apoptosis, cell proliferation/differentiation, inflammation, chromatin biology, and cell signalling.,

Phospho-specific antibodies, with no cross-reactivity to other phosphoamino acids or the non-phosphorylated residue,

\footnotetext{
${ }^{a}$ Department of Chemistry, The University of Sheffield, Dainton Building, Sheffield, S3 7HF, UK. E-mail: r.f.w.jackson@shef.ac.uk

${ }^{b}$ Department of Infection and Immunity, Academic Unit of Respiratory Medicine, The University of Sheffield Medical School, Beech Hill Road, Sheffield, S10 2TH, UK. E-mail: r.muimo@shef.ac.uk

$\dagger$ Electronic supplementary information (ESI) available: Experimental procedures and NMR spectra for 2, 3, 5, 6, 7 and 8. See DOI: $10.1039 / \mathrm{c} 5 \mathrm{cc} 01811 \mathrm{k}$
}

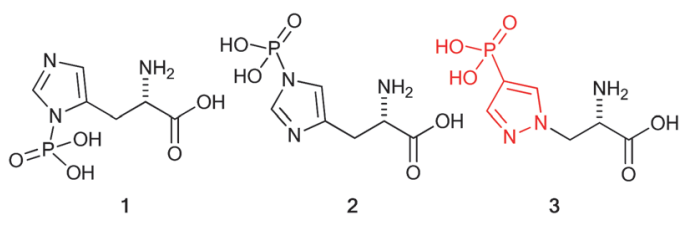

Fig. 1 Isomeric forms of phosphohistidine and the stable mimic, 4-phosphopyrazol-2-yl alanine (pPza).

have played a key role in elucidating the importance of $O$-phosphorylation in cell signalling. For example, the generation of phosphotyrosine specific antibodies in the early 1980s led to a massive upsurge in phosphotyrosine research and elucidation of the role of this posttranslational modification in many key cellular processes. $\tau$-Phosphohistidine specific antibodies would likewise be pivotal in understanding the precise roles of histidine phosphorylation, but phosphohistidine itself is a poor immunogen because of its high susceptibility to hydrolysis ( $\Delta G^{\circ}$ of hydrolysis -50 to $-59 \mathrm{~kJ} \mathrm{~mol}^{-1}$ ), and cannot be used to generate specific antibodies.

An alternative strategy is to produce 'phosphohistidine-like' antibodies by using stable unnatural amino acid analogues or mimics that are immunogenic. Since the discovery of phosphohistidine, only a relatively small number of potential analogues have been developed including thio-phosphohistidine, ${ }^{7}$ phosphofurylalanine, ${ }^{8}$ phosphopyrrole, ${ }^{5}$ phosphoryltriazolylalanine isomers, ${ }^{9-11}$ and most recently phosphoryltriazolylethylamine. ${ }^{12}$ A sulphonamide mimic of the transition state of phosphohistidine phosphatase has been reported, ${ }^{13}$ as has the synthesis of a complementary triazole. ${ }^{14}$ In most of the stable mimics, the labile $\mathrm{P}-\mathrm{N}$ bond is replaced with a hydrolytically stable $\mathrm{P}-\mathrm{C}$ bond. Introduction of a $\mathrm{P}-\mathrm{C}$ bond was very successful in the case of phosphotyrosine ${ }^{15}$ which is also relatively unstable. In one early report of a phosphotyrosine antibody generated using azobenzene phosphonate as immunogen, Frackelton et al. observed crossreactivity towards phosphohistidine $3,{ }^{16}$ and thus provided the first indication that it might be possible to raise antibodies specific to phosphohistidine. However, antibodies subsequently raised against 
the various analogues outlined above either cross-reacted significantly with other phosphorylated amino acids; did not universally recognise phosphohistidine in proteins; or did not recognise it at all. Consequently, the study of $N$-phosphorylation in cell biology has remained in its infancy, especially in eukaryotes, despite the fact that phosphohistidine was discovered nearly 20 years prior to phosphotyrosine and is reported to be much more abundant than phosphotyrosine in certain tissues and organisms. ${ }^{17,18}$

Motivated by a previous report that suggested phosphofurylalanine ${ }^{8}$ might be a suitable non-hydrolysable and non-isomerisable analogue of $\tau$-phosphohistidine 2 , we considered that the simplest modification would involve transposition of the nitrogen atom bound to the labile phosphoryl group and the carbon bound to the alanine residue. Pyrazole 3 therefore became our initial target. ${ }^{19}$ The synthesis of $\mathbf{3}$ was carried out by ring-opening of the Vederas $\beta$-lactone $4^{20-22}$ with 4 -iodopyrazole to give the acid 5 . Formation of the methyl ester 6 and subsequent Hirao cross-coupling ${ }^{23}$ with diethyl phosphite gave the fully protected amino acid 7. Finally twostage deprotection was carried out by hydrolysis of the methyl ester to give the acid $\mathbf{8}$, followed by treatment with TMSBr to give the target, 4-phosphopyrazol-2-yl alanine (pPza) 3 (Scheme 1).

To test the suitability of pPza 3 as an analogue of $\tau$-phosphohistidine, it was coupled to keyhole limpet hemocyanin (KLH) via glutaraldehyde linkage and used as a hapten to generate rat and rabbit polyclonal antibodies. The resulting antisera was tested for specificity using dot blot analysis (Fig. 2) against amino acid standards conjugated to bovine serum albumin (BSA), also via glutaraldehyde linkage. The polyclonal antibodies generated from pPza 3 showed strong reactivity towards $\tau$-phosphohistidine, minor reactivity to histidine, and no cross-reactivity towards phosphotyrosine or other $\mathrm{O}$-phosphoamino acids (Fig. 2, entry A). Crossreactivity towards histidine was removable by affinity depletion using a histidine-KLH glutaraldehyde conjugated sepharose column (Fig. 2, entry C) but not a histidine sepharose column (Fig. 2, entry B) as previously described. ${ }^{24}$ The $O$-phosphoamino

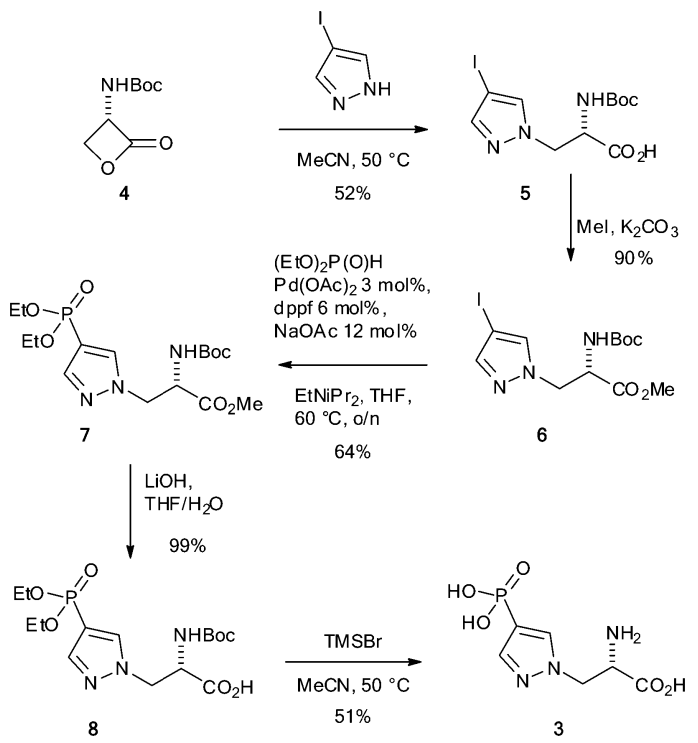

Scheme 1 Synthesis of 4-phosphopyrazol-2-yl alanine 3.

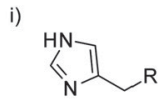

ii)

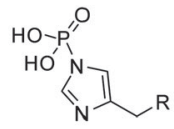

iv)

v)

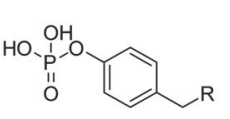

iii)<smiles>[R]COP(=O)(O)O</smiles>

vi)

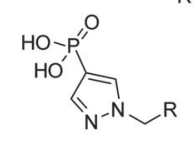

$R=\overbrace{\prod_{O}}^{N_{B} H_{2}} \mathrm{OH}$

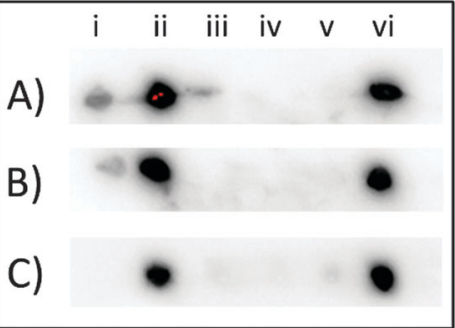

Fig. 2 Dot-blots obtained using pPza rat antisera: (A) crude polyclonal antisera. (B) Antibody affinity depleted using a sepharose histidine column. (C) Antibody affinity depleted with sepharose bound glutaraldehyde linked histidine-KLH column. (i) Histidine, (ii) $\tau$-phosphohistidine, (iii) phosphoserine, (iv) phosphothreonine, (v) phosphotyrosine, (vi) 4-phosphopyrazol2-yl alanine (pPza).

acid standards (Fig. 2; iii-v), linked via succinylated BSA, were commercially sourced. We have already shown that these standards are detected by an antibody generated to a glutaraldehyde-linked antigen, thus demonstrating that the absence of cross-reactivity towards $O$-phosphoamino acids was not a false negative. ${ }^{24}$

Consequently, a two stage strategy was adopted to purify the rabbit pHis antibody: firstly, antisera was affinity depleted using an agarose column containing histidine conjugated to BSA via glutaraldehyde linker (see ESI, $\dagger$ Fig. S1); secondly, the flow through from the first column was then further purified on an affinity column containing immobilized BSA- $\tau$-phosphohistidine conjugate. ELISA analysis showed the purified antibody had high specificity for $\mathrm{pHis}$ with little or no cross-reactivity for pTyr or other phosphoamino acids tested (Fig. 3).

Western blots were performed with $\tau$-phosphohistidine-BSA conjugate to establish the utility of the antibody in a standard biological technique (see ESI, $\uparrow$ Fig. S3). Further western blot analysis was also performed against an extract of cytosolic proteins from ovine airway epithelia treated with either acid, base or hydroxylamine buffers as previously described. ${ }^{25}$ Protein bands (as yet unidentified) detected by the pPza rabbit antibody are stable under alkaline conditions but labile in the presence of hydroxylamine or acidic conditions (Fig. 4A), indicative of a phosphoramidate bond. Lysates of human bronchial epithelial cells (16HBE140-) showed a considerable increase in phosphotyrosine content in several protein bands following treatment with human epidermal growth factor (EGF, $15 \mathrm{nM}$ ) for $60 \mathrm{~min}$ at $37{ }^{\circ} \mathrm{C}$ in full growth medium (Fig. 4B, left panel), but this greatly increased level of phosphotyrosine in the EGF treated cells remained undetected by the pPza antibody (Fig. 4B, right panel). These data provide evidence that in a complex protein sample, the pPza antibody specifically detects phosphoramidate or $N$-phosphorylation and not acyl or $O$-phosphoamino acids. 


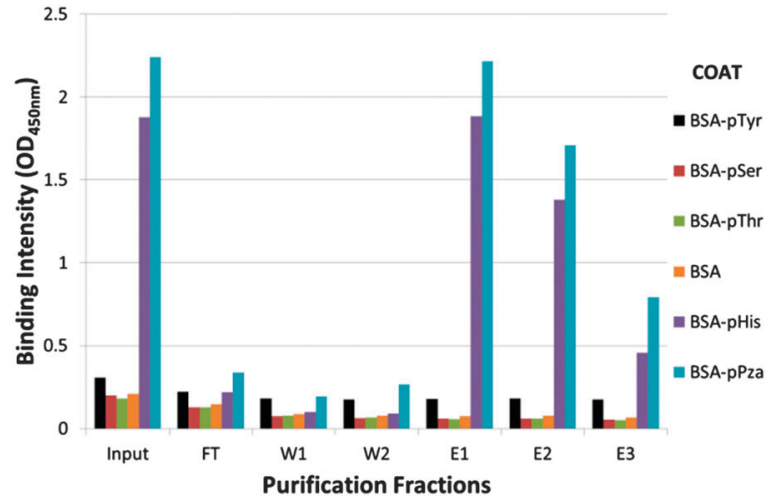

Fig. 3 Extract from the affinity purification of pPza rabbit antisera. Rabbit polyclonal antibody affinity depleted using an agarose-BSA-His affinity column was purified on an agarose-BSA-pHis affinity column: ELISA analysis showing binding intenstity of pPza antibody to phosphoamino acids in various purification fractions measured at $450 \mathrm{~nm}$ - input, flow through (FT), washes and eluates. The ELISA plate was coated with BSA, BSA-His, BSA-pHis, BSA-pThr, BSA-pSer, BSA-pTyr or BSA-pPZa. The full purification profile is included in the ESI, $\dagger$ Fig. S2.
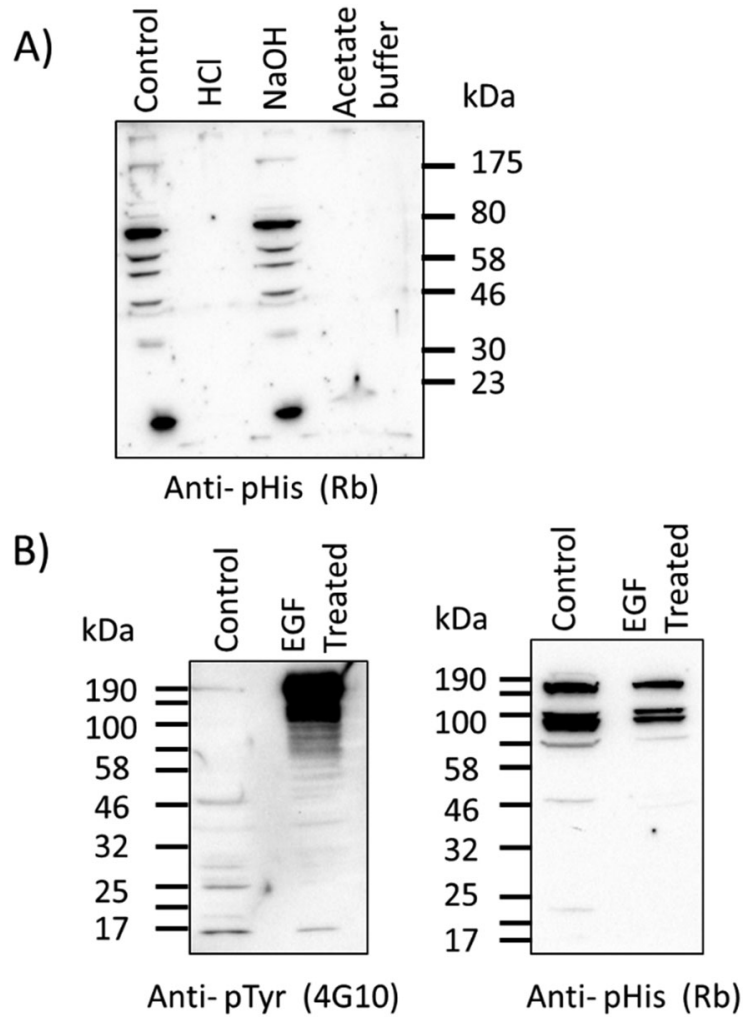

Fig. 4 Western blot detection of phosphohistidine (anti-pHis) and phosphotyrosine (anti-pTyr) following EGF, acid or base treatments. (A) Western blot of cytosolic proteins from ovine airway epithelia treated with buffer control, $0.1 \mathrm{M} \mathrm{HCl}, 0.1 \mathrm{M} \mathrm{NaOH}, 0.4 \mathrm{M}$ acetic acid/0.1 M hydroxylamine, for $30 \mathrm{~min}$ at $60{ }^{\circ} \mathrm{C}$, and probed for phosphohistidine (pPza rabbit antibody, $\mathrm{Rb})$. (B) Lysates of human bronchial epithelial cell lines (16HBE14o-) treated with or without human epidermal growth factor (EGF, $15 \mathrm{nM}$ ) for 1 hour and probed for phosphotyrosine staining (clone 4G10 antibody) or the rabbit antibody generated with pPza (right panel), (control, untreated).

A pan-pHis specific antibody that recognises pHis epitopes independently of sequence context should allow the detection

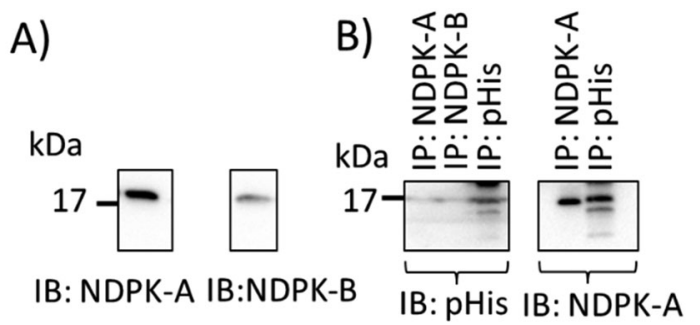

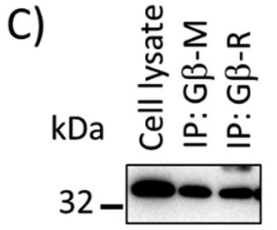

IB: Protein G $\beta$

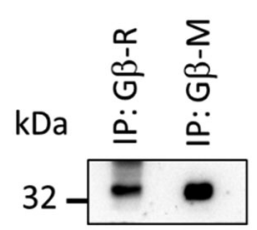

IB: $\mathrm{pHis}$
Fig. 5 Detection of endogenous phosphohistidine in known phosphohistidine containing proteins (NDPK-A or B, and protein G $\beta$ from airway epithelia). (A) Immunoblot (IB) of NDPK-A and NDPK-B; (B) western blot detection of phosphohistidine and NDPK-A in immunoprecipitates (IP) of NDPK-A, NDPK-B and phosphohistidine (pHis) from cytosol of ovine airway epithelia or (C) western blot detection of phosphohistidine in immunoprecipitates (IP) of protein G $\beta$ (antibodies $\mathrm{R}=$ rabbit polyclonal; $\mathrm{M}=$ mouse monoclonal) in lysates of human bronchial epithelial cells (16HBE14o-) probed with pPza rabbit antibody.

of many histidine-phosphorylated proteins and be useful in interrogation of proteomes. We therefore sought to provide further evidence that the pPza antibody recognises phosphohistidine independently of the amino acid sequences flanking the histidine phosphorylation sites. Fig. 5 shows immunoblot detection of endogenous phosphohistidine in different proteins - NDPK-A, NDPK-B, and protein $G \beta^{26,27}$ - immunoprecipitated from lysates of human bronchial epithelial cells (16HBE140-) and further demonstrates the potential and wide-ranging utility of the pPza generated antibody to detect phosphohistidine in a range of proteins. As expected, heat treatment of the immunoprecipitates at $90{ }^{\circ} \mathrm{C}$ for $5 \mathrm{~min}$ in Laemmli buffer resulted in loss of pHis signal (data not shown).

Significantly, the pPza generated antibody selectively recognizes free $\tau$-phosphohistidine, detects phosphorylated histidine residues in several proteins and shows little or no cross-reactivity with $O$-phosphoamino acids.

In conclusion, we report successful synthesis of 4-phosphopyrazol2-ylalanine 3 and demonstrate its potential as a stable analogue of $\tau$-phosphohistidine. $\ddagger$ Thus, both pPza and resulting antibodies are likely to be important tools to explore and characterize the role of phosphohistidine in various cell types and cellular processes.

We thank the EPSRC Life Sciences Interface DTC for support (ML), and B. Marsh for experimental assistance. The 16HBE140cells were provided by Dr D. Gruenert (California Pacific Medical Center, CA).

\section{Notes and references}

$\ddagger$ During the preparation of this manuscript, the synthesis of a closely related pyrazolylethylamine derivative, and its use to generate antibodies, was reported. $^{28}$ These antibodies showed significantly higher selectivity towards phosphohistidine (compared to other $O$-phosphoamino acids) than antibodies generated from the previously reported triazole analogues. 
1 T. Hunter, Philos. Trans. R. Soc. London, Ser. B, 2012, 367, 2513-2516.

2 P. Cohen, Trends Biochem. Sci., 2000, 25, 596-601.

3 O. Zetterqvist and L. Engstrom, Biochim. Biophys. Acta, 1966, 113, $520-530$.

4 P. D. Boyer, Science, 1963, 141, 1147-1153.

5 P. V. Attwood, M. J. Piggott, X. L. Zu and P. G. Besant, Amino Acids, 2007, 32, 145-156.

6 J.-M. Kee and T. W. Muir, ACS Chem. Biol., 2012, 7, 44-51.

7 M. C. Pirrung, K. D. James and V. S. Rana, J. Org. Chem., 2000, 65, 8448-8453.

8 C. Schenkels, B. Erni and J. L. Reymond, Bioorg. Med. Chem. Lett., 1999, 9, 1443-1446.

9 J.-M. Kee, B. Villani, L. R. Carpenter and T. W. Muir, J. Am. Chem. Soc., 2010, 132, 14327-14329.

10 T. E. McAllister, M. G. Nix and M. E. Webb, Chem. Commun., 2011, 47, 1297-1299.

11 S. Mukai, G. R. Flematti, L. T. Byrne, P. G. Besant, P. V. Attwood and M. J. Piggott, Amino Acids, 2012, 43, 857-874.

12 J.-M. Kee, R. C. Oslund, D. H. Perlman and T. W. Muir, Nat. Chem. Biol., 2013, 9, 416-421.

13 M. F. Eerland and C. Hedberg, J. Org. Chem., 2012, 77, 2047-2052.

14 S. H. Yang, D. J. Lee and M. A. Brimble, Org. Lett., 2011, 13, 5604-5607.

15 M. K. Tarrant and P. A. Cole, Annu. Rev. Biochem., 2009, 78, 797-825.
16 A. R. Frackelton, A. H. Ross and H. N. Eisen, Mol. Cell. Biol., 1983, 3, 1343-1352.

17 K. H. Pesis, Y. Wei, M. Lewis and H. R. Matthews, FEBS Lett., 1988, 239, 151-154.

18 L. L. Bieber and P. D. Boyer, J. Biol. Chem., 1966, 241, 5375-5383.

19 R. Jackson, R. Muimo and M. Lilley, PCT application pending, WO2015/033120 A1, PCT/GB2014/052646, 2014.

20 L. D. Arnold, T. H. Kalantar and J. C. Vederas, J. Am. Chem. Soc., 1985, 107, 7105-7109.

21 S. V. Pansare, L. D. Arnold and J. C. Vederas, Org. Synth., 1991, 70, 10-17.

22 S. V. Pansare, G. Huyer, L. D. Arnold and J. C. Vederas, Org. Synth., 1991, 70, 1-9.

23 M. Kalek, M. Jezowska and J. Stawinski, Adv. Synth. Catal., 2009, 351, 3207-3216.

24 M. Lilley, B. Mambwe, R. F. W. Jackson and R. Muimo, Chem. Commun., 2014, 50, 9343-9345.

25 R. Muimo, Z. Hornickova, C. E. Riemen, V. Gerke, H. Matthews and A. Mehta, J. Biol. Chem., 2000, 275, 36632-36636.

26 P. V. Attwood and T. Wieland, Naunyn-Schmiedeberg's Arch. Pharmacol., 2015, 388, 153-160.

27 R. Muimo, S. J. Banner, L. J. Marshall and A. Mehta, Am. J. Respir. Cell Mol. Biol., 1998, 18, 270-278.

28 J.-M. Kee, R. C. Oslund, A. D. Couvillon and T. W. Muir, Org. Lett., 2015, 17, 187-189. 